Editorial

Z Herz- Thorax- Gefäßchir 2019 · 33:375

https://doi.org/10.1007/s00398-019-00342-5

(c) Springer Medizin Verlag GmbH, ein Teil von Springer Nature 2019

Liebe Leserinnen und Leser der Zeitschrift für Herz-, Thorax- und Gefäßchirurgie,

in dieser aktuellen Ausgabe der Zeitschrift werden Sie verwöhnt!

Herr Prof. Doenst und sein Team aus Jena versorgen Sie mit zwei Übersichten zur aktuellen Literatur mit Bezug zu unserem Fachgebiet: Die „Literaturübersicht 2018 zur Herzklappenchirurgie“ und die „Literaturübersicht 2018 zur Koronarchirurgie“. Einfacher geht es für Sie nicht, wenn Sie die aktuelle Studienlage zu diesen beiden quantitativ wichtigsten Bereichen unseres Fachgebietes kennen wollen: Gut strukturiert, inhaltlich geordnet werden in beiden Beiträgen die wichtigsten offenen Fragen mit der aktuellen Literatur diskutiert und z.T. beantwortet. Zunächst ist man etwas erschlagen ob der vielen Daten und Zahlen, aber diese dienen nur dem Zweck, die erarbeiten Aussagen, die sich aus den zitierten Publikationen ergeben, zu belegen. Bequemer geht es für den Leser kaum: keine zeitaufwendige Medline-Suche, keine mühsame Gewichtung zahlreicher Studien und die Suche nach der neuen Erkenntnis, sondern auf dem Silberteller mundgerecht servierte Fakten.

So werden wichtige Fragen wie die Relevanz geringgradiger perivalvulärer Insuffizienzen nach TAVI beantwortet (...schon ein geringgradiges PVL ist ein unabhängiger Risikofaktor für das Fünfjahresüberleben im US CoreValve Trial) und erste Tendenzen im mittleren Langzeitverlauf dargestellt (... ebenfalls US CoreValve Trial: Der initial beobachtete Überlebensvorteil der TAVI gegenüber dem Aortenklappenersatz ist nach 5 Jahren aufgebraucht). Das sollte nicht nur der Herzchirurg wissen, wird doch der US CoreValve Trial immer wieder als

\author{
K. Kallenbach \\ Haerzzenter - INCCI, Luxembourg, Luxemburg
}

\title{
Lassen Sie sich verwöhnen!
}

Rechtfertigung der TAVI im Niedrigrisikobereich herangezogen. Auch für die Wahl der geeigneten Aortenklappenprothese bei der offenen Operation tut sich etwas: Einige mechanische Klappenprothesen lassen sich mittlerweile mit einem INR kleiner 2,0 sicher behandeln, was einen hohen Stellenwert in der Vermeidung von Blutungskomplikationen unter der Marcumar-Therapie einnimmt. Dieses Ergebnis könnte Einfluss auf die gängige Praxis der Prothesenwahl beim Aortenklappenersatz nehmen: Vielleicht wird so die aus meiner Sicht ausufernde Verwendung von biologischen Klappenprothesen bei Patienten unter 60 Jahren, die wissenschaftlich nicht $\mathrm{zu}$ begründen ist, ein wenig zurückgenommen. Lesen Sie den Beitrag, und Sie werden viele neue und wichtige Erkenntnisse gewinnen - da bin ich mir sicher!

Auch in der Koronarchirurgie tut sich (immer wieder) etwas: Dass die chirurgische koronare Revaskularisation als einzige Therapieform das Potenzial besäße, das Leben zu verlängern, schlussfolgern die Autoren aus den aktuell verfügbaren Langzeitdaten im Vergleich von PCI vs. ACVB. Sehr bemerkenswert sind für mich 2 Beobachtungen: 1. Drei Studien konnten nachweisen, dass die ACVB-Operation im Vergleich zur PCI zu deutlich besserem Überleben bei Patienten mit reduzierter Pumpfunktion (EF <35\%) führt. Hier vermutet das Autorenteam einen revaskularisationsunabhängigen Effekt, die „chirurgische Kollateralisierung“. Tatsächlich wird in der klinischen Praxis gerade die schlechte Pumpfunktion als Argument pro PCI angeführt. 2. In der SYNTAX-III-REVOLUTION-Studie wurde quasi eine Gleichwertigkeit des Koronar-CT mit der Koronarangiographie gezeigt. Sollten sich diese Daten bestätigen, was bei der fortschreitenden Entwicklung der CT-Technik zu erwarten ist, wird die invasive Koronardiagnostik obsolet, und die Entscheidung der besten Revaskularisationsstrategie - ACVB vs. PCI kann tatsächlich ohne vorherige Bahnung gemeinsam im Herzteam getroffen werden.

Natürlich handelt es sich hier um eine Selektion der verfügbaren Literatur, und natürlich hat das Autorenteam eine $\mathrm{Ge}$ wichtung vorgenommen. Ein Anspruch auf neutrale Vollständigkeit besteht also nicht, aber diese zwei Beiträge helfen Ihnen, in der Diskussion um die geeignete Therapie Argumente parat zu haben.

Viel Spaß beim „Sich-verwöhnenLassen“,

Ihr

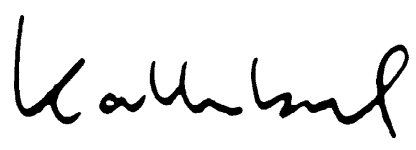

Klaus Kallenbach

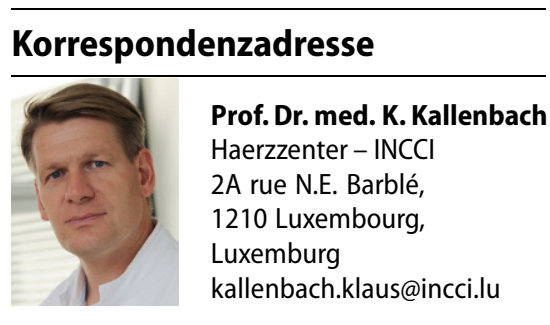

Interessenkonflikt. K. Kallenbach gibt an, dass kein Interessenkonflikt besteht. 